\title{
Side Effects of Herbal Drugs Used in Dermatologic Disorders
}

\author{
Gita Faghihi, Mohammadreza Radan
}

Dermatology department, Isfahan University of Medical sciences, Isfahan, Iran; Councelor of Medical Practice Supervision , Isfahan University of Medical sciences, ,Isfahan, Iran

Email: g_faghihi@med.mui.ac.ir

Received January $25^{\text {th }}, 2011$; revised February $3^{\text {rd }}, 2011$; accepted February $11^{\text {th }}, 2011$.

\begin{abstract}
Because of the awareness of the environmental damage caused by industrialization, a trend has developed to use products with natural ingredients. Herbal products have been used for the treatment of disease, most commonly in the last century. As herbal supplements become more popular, several adverse side effects have been reported in users who also take other medications. In dermatology, many botanic products are used especially with regard to cosmetic benefits of natural topical products on skin aging process, so dermatologists should face with higher side effects of these types of drugs. The most common presentation of topical aromatherapy side effects are contact dermatitis and the second in order will be contact urticaria reactions.
\end{abstract}

Keywords: Herbs, Dermatology, Cutaneous, Side Effects

\section{Back ground}

Many patients with chronic dermatological diseases have attempted to take more control over their health by using herbal remedies along with or instead of conventional treatments. Some patients have lost hope; standard treatments have failed to be effective for them. As a result, they seek newer therapies in an attempt to find a "cure" for their problems [1].

Because of the awareness of the environmental damage caused by industrialization, a trend has developed to use products with natural ingredients [2]. Herbal products have been used for the treatment of disease, most commonly in the last century. As herbal supplements become more popular, several adverse side effects have been reported in users who also take other medications [3].

\section{Examples of Botanicals Used in Dermatology and Cosmetic Skin Practice:}

\subsection{Avocado and Chamomile}

Which inhibit the release of histamine and has antiinflammatory properties. Chamomile has also antibacterial, anti-inflammatory and fungicidal effects which will be used in atopic dermatitis, Candida albicans and gram-positive infections [4].

\subsection{Ginseng}

Which stimulates the biosynthesis of proteins, RNA and lipids [5].

\subsection{Ginkgo Biloba Extract}

Induce SOD and catalase enzyme in the epidermis after topical application as well as to systemically increase the activity of both enzymes in the liver, the heart and kidneys [6].

\subsection{Glycyrrhizin}

In licorice roots inhibits pro-inflammatory activities of prostaglandins and leukotrienes [7].

\subsection{Capsaicin}

Inhibits substance $\mathrm{P}$, a peptide transmitter of the inflammatory process [8].

\subsection{Aloe vera}

Accelerate wound healing and to protect and sooth the skin [9].

\section{Range of Cutaneous Side Effects}

The most common dermatologic reaction from herbal 
therapies is allergic contact dermatitis.

Herbs that are known for causing this condition include: aloe, arnica, bromelain, calendula, chamomile, goldenseal, tea tree oil and yarrow [1]. However, more serious events have occurred including erythroderma and Stevens-Johnson syndrome from combination herbal preparations. Serious systemic adverse events have been reported with herbal therapies for the treatment of dermatological diseases as well [10]. Most are hepatotoxic effects and some have been fatal although this is rare [11].

Herbs recommended for topical use should not be ingested, vice-versa. Drug interactions that most commonly occur are due to immuneomodulatory reactions, however effects on anticonvulsants and anticoagulants can occur [12]. We observed a case of severe toxic irritant dermatitis from watercress in a 60 years old female.

Celery is known to contain psoralens, a group of substances that cause a toxic dermal reaction on exposure to ultraviolet A rays .Celery may increase the risk of bleeding or potentiate the effects of warfarin therapy. Celery root is a frequent cause of food allergy in pollen-sensitized patients [13].

\subsection{Classification of the Common Herbs used in Dermatology}

\subsubsection{Anti - Bacterial Agents}

They are diversely used in acne, impetigo and pyodermas. Tea tree oil is one of popular ones. It can cause allergic contact dermatitis [8].

\subsubsection{Anti - Fungals}

Henna causes severe allergic reactions, even some case reports of systemic anaphylaxis were reported [14]. Chamomile with several anti-fungal and anti-microbial properties may induce hypersensitivity cross-reactions to ragweed, Chrysanthemums (Compositae family) [15].

\subsubsection{Furocoumarine Containing Herbs}

Parsley, carrots, trigoneum, lemon, Bergamots may cause severe photo toxicity and post burning - pigmentary changes in the skin [16].

\subsubsection{Odorous Compounds}

Propolis, balsams and essential oils have the potential to initiate allergic reactions due to their volatile and skin absorbent nature [17].

\subsubsection{Weight Loss Products}

Ephedra has been linked to deaths, strokes, heart attacks and other health problems. Ephedra is like amphetamines in that it stimulates the central nervous system and is used in many weight loss and increased energy products. Green Tea known for its antioxidant and weight control- ling abilities has been associated with documented reports of potential interactions with warfarin. [13,14].

\subsubsection{Skin Soothing and Healing AgentsExamples as} follow:

Evening primrose oil which has several Side effects like nausea (may decrease if taken with food), skin rashes and acne [18]. Comfrey that is widely used for a variety of conditions particularly as a wound healing agent topically will be uNsafe for internal use and perhaps, topically for deep wounds because it contains hepatotoxic alkaloids.

Aloe vera's gel (juice) is for minor burns, abrasions and irritations on topical application. The gel may inhibit bradykinin and hinders the formation of thromboxan. It should not be used in deep vertical cuts because it may delay healing [9]. In some people it causes allergic dermatitis.

\subsubsection{Herbs with Hormonal Side Effects}

Ginseng causes diminished platelet adhesivness, so monitor with anticoagulants. It may potentiate MAO inhibitors and should not be used with stimulants, antipsychotic drugs and hormone treatment [14].

It should not be used in diabetics because of hypoglycemic effect may cause breast tenderness in women and nervousness. Women may experience estrogenic side effects [5]. Sage is also responsible for irregular menses and breast tenderness. Fenugreek causes hypoglycemia and licorice should not be used in hypertension and induces hypokalemia as an aldosterone-like effect [4].

\section{Protocols of Safety Usage}

Many of these therapies are considered "natural" and therefore harmless. However, because of the poor regulations that exist in monitoring these drugs, adverse reactions do occur. Phytotherapy (treatment with herbs), therefore, should be avoided in pregnancy, infants and children because of the uncertainty of adverse reactions that could occur [18,19].

\section{Conclusion}

A brief search of the literature reveals many therapies used for dermatological disease however there are fortunately fewer reports of their side effects in .It is important for dermatologists to become aware of these adverse events and interactions in order to better educate their patients and possibly prevent potential and unexpected adverse reactions.

\section{REFERENCES}

[1] M. K. Bedi and P. D. Shenefelt, "Herbal Therapy in Dermatology," Archives of Dermatology, Vol. 138, No. 2, 
2002, pp. 232-242. doi:10.1001/archderm.138.2.232

[2] R. B. Vender, "Adverse reactions to herbal therapy in dermatology," Skin Therapy Letter, Vol. 8, No. 3, 2003, pp. 5-8

[3] K. H. Neldner, "Complementary and Alternative Medicine," Dermatology Clinics, Vol. 18, No. 1, 2000, pp. 189-193. doi:10.1016/S0733-8635(05)70159-5

[4] J. Graf, "Herbal anti-inflammatory agents for skin disease," Skin Therapy Letter, Vol. 5, No. 4, 2000, pp. 3-5.

[5] M. H. Rustin and L. Poulter, "Chinese Herbal Therapy in atopic dermatitis," Dermatol Ther, Vol. 1, No. 1, 1996, pp. 83-93.

[6] P. Gardiner and K. J. Kemper, "Herbs in pediatric and adolescent medicine," Pediatrics in Review, Vol. 21, No. 2, 2000, pp. 44-57. doi:10.1542/pir.21-2-44

[7] M.K. Bedi and P. D. Shenefelt, "Herbal Therapy in Dermatology," Archives of Dermatology, Vol. 138, No. 2, 2002, pp. 232-242.doi:10.1001/archderm.138.2.232

[8] R. Norman and D. Nelson, "Do Alternative \& Complementary Therapies work for common Dermatologic Conditions," Skin \& Aging, Vol. 8, 2000, pp. 28-33.

[9] D. Mantle, M. A. Gok and T. W. Lennard, "Adverse and beneficial effects of plant extracts on skin and skin disorders," Adverse Drug Reaction Toxicology Revew, Vol. 20, No. 2, 2001, pp. 89-103.

[10] F. M. Keane, A. W. du Vivier, et al, "Analysis of Chinese herbal creams prescribed for dermatological conditions," British Medical Journal, Vol. 318, No. 7183, 1999, pp. 563-4.

[11] D. S. Sitar, "Important Drug Interactions," The Canadian
Journal of CME, 2002, pp: 77-87.

[12] E. Ernst, "Adverse effects of herbal drugs in dermatology," British Journal of Dermatology, Vol. 143, No. 5, 2000, pp. 923-929.

doi:10.1046/j.1365-2133.2000.03822.x

[13] A. M. Heck, B. A. DeWitt and A. L. Lukes, "Potential interactions between alternative therapies and warfarin," American Journal of Health-System Pharmacy, Vol. 57, No. 13, 2000, pp. 1221-1227

[14] G. A. Wong and N. H. Shear, "Adverse drug interactions and reactions in dermatology: current issues of clinical relevance," Dermatology Clinics, Vol. 23, No. 2, pp. 335-42.F. Giordano-Labadie, H. P. Schwarze and J. Buzex, "Allergic contact dermatitis from camomile used in phytotherapy," Contact Dermatitis, Vol. 42, No. 4, 2000, p. 247

[16] L. A. Gordon, "Compositae dermatitis," Australasian Journal of Dermatology, Vol. 40, No. 3, 1999, pp. 123-128, quiz. 129-130.

doi:10.1046/j.1440-0960.1999.00341.x

[17] P. T. Ting and S. Silver, "Allergic contact dermatitis to propolis," Journal of Drugs in Dermatology Articles, Vol. 3, No. 6, 2004, pp. 685-686.M. R. Buchness, "Alternative medicine and dermatology," Seminars in Cutaneous Medicine Surgery, Vol. 17, No. 4, 1998, pp. 284-290. doi:10.1016/S1085-5629(98)80025-4

[19] M. Neuman, "Metabolic effects and drug interactions provoked by certain vegetables: grapefruit, St. John's wort and garlic," Press Med, Vol. 31, No. 30, 2002, pp. 1416-1422. 